\title{
ESTUDIO DE CARACTERIZACIÓN DEL ÁREA TELEINFORMÁTICA
}

Miguel Angel Vargas Hernández ${ }^{*}$ Eugenio Paniagua Suarez ${ }^{* *}$

\begin{abstract}
Objectives and Scope of this Study

The objective of the study is to describe and evaluate the economical, organizational, occupational and educational environments of the Area of Tele-information technology in Colombia. This is, to identify them on national scale and define their nature, characteristics and trends, emphasizing the identification of the significant links among the occupational groups that conform them.
\end{abstract}

\section{Methodology}

\section{Population}

Three populations have been considered for this study, among which the information was collected. The populations are: the companies' users of teleinformation technology, the suppliers of information technology solutions, and the institutions of professional technical and technological formation and training.

\section{Plan for the Analysis}

Twenty-nine secondary sources were consulted and a survey was carried out, directed to IT and communications suppliers and user companies, besides the IT and communication programs offered by the universities and institutions of post -secondary education.

In order to analyze data and synthesize the information, a Factorial Analysis of Multiple Correspondence was made (ACM).

\section{Results}

Five critical factors were identified in the Area of tele-Information technology, from which its development depends:

6. Marketing

7. Research and Development.

8. Contracting Procedures.

9. Professionals and technologists with a high level of formation and experience in management of tele-IT projects and programming.

10. Quality Culture.

\footnotetext{
${ }^{*}$ Natjonal Executiee Presjdent of the Colombian Association for informatjon Technology and Communications - ACUC, academic coordinator of the Communication - Education program of the Research Departmenf of the Unjversjdad Central, DIUC. Graduated in Education with specialty in Educational IT, Speoialist in Software Engineering with emphasis in Organizational and Systems Consultjng from the UIS. Profesor Universidad de Antioquia. Matemático, especialista en Estadística - Universidad Nacional. Especialista en Ingeniería del Software - UIS.
} 


\section{Conclusions}

More than $90 \%$ of the companies consider that very few pro fessionals from the Area of Tele-IT are capable of performing a demanding profession.

In general, Tele-IT is more important as a strategic business area for the universities than as a field for research, study and professional formation.

\section{Key words}

Characterization of tele-information technology: Systematic Process for the description and evaluation of the economical, organizational, occupational, and educational environments of the user and provider companies of information technologies.

Information Technology: Branch of technology that deals with the information and knowledge and manages them as technological objects.

Computational infrastructure: Technological objects with capacity to receive, manipulate, and store digital information by means of computers and Internet.

Information Infrastructure: Technological objects capable of receiving and sending information in voice, text, and video formats.

Social Infrastructure: Capacity of people to take advantage of information and Communication Technologies.

\section{RESUMEN}

\section{Objetivos y alcance del estudio}

El estudio tiene como objetivo describir y evaluar los entornos: económico, organizacional, ocupacional y educativo del área de la teleinformática en Colombia. Esto es, identificarlos a escala nacional, y definir su naturaleza, características y tendencias haciendo énfasis en la identificación de los enlaces significativos entre los grupos ocupacionales que lo conforman

\section{Metodología}

\section{Población}

Para el estudio se cuenta con tres poblaciones dentro de las cuales se recogió la información, que son: las empresas usuarias de la teleinformática, los proveedores de soluciones informáticas y las instituciones de formación y capacitación profesional, técnica y tecnológica.

\section{Plan de análisis}

Se consultaron 29 fuentes secundarias y se realizó una encuesta dirigida a los empresarios de la informática y las comunicaciones, empresas usuarias, además de los programas de informática y comunicaciones que ofrecen las universidades e instituciones de educación possecundaria 
Para analizar datos y sintetizar información, se hizo un Análisis Factorial de Correspondencia Múltiple, ACM.

\section{Resultados}

En el área de la teleinformática se identifican cinco factores críticos, de los cuales depende su desarrollo:

1. Mercadeo

2. Investigación y desarrollo.

3. Procesos de contratación.

4. Profesionales y tecnólogos con alto nivel de formación y experiencia en gerencia de proyectos teleinformáticos y programación.

5. Cultura de la calidad.

\section{Conclusiones}

Más del $90 \%$ de los empresarios consideran que muy pocos profesionales del área de la teleinformática están capacitados para ejercer una profesión exigente.

En general, para las universidades es más importante la teleinformática como un área estratégica de negocio que como un campo de investigación, estudio y formación profesional.

\section{Palabras clave}

Caracterización teleinformática: proceso sistemático de descripción y evaluación de los entornos económico, organizacional, ocupacional y educativo de las empresas usuarias y proveedoras de tecnologías informáticas y telemáticas.

Informática: rama de la tecnología que trata de la información y el conocimiento y los maneja como objetos tecnológicos.

Infraestructura computacional: objetos tecnológicos con capacidad para recibir, manipular y almacenar información digital a través de computadores y el Internet.

Infraestructura de información: objetos tecnológicos que tiene la capacidad de recibir y enviar información en formatos de voz, texto y vídeo.

Infraestructura social: capacidad de las personas para aprovechar las tecnologías de la información y la comunicación.

\section{Diseño del estudio}

\section{Poblaciones}

Para el estudio sobre la caracterización ocupacional y educativa del área teleinformática, se cuenta con tres poblaciones dentro de las cuales se recogió la información, que son: las empresas usuarias de la tele informática, los proveedores de soluciones informáticas y las instituciones de formación y capacitación en la teleinformática.

Empresas usuarias es una muestra aleatoria de acuerdo a la clasificación que tiene Confecámaras por actividad económica y por ciudad, para lo cual se hace una asignación 
proporcional de la muestra de las empresas de acuerdo al número que haya en cada ciudad. La forma de recolección de la información fue mediante una encuesta enviada por correo.

La unidad de análisis tuvieron base en las empresas usuarias y la información la proporcionarán los directores o jefes de sistemas.

Empresas proveedoras: se hizo una clasificación por actividad, dividiéndolas en dos estratos: proveedores de software y servicios asociados, proveedores de hardware.

La unidad de análisis es la empresa, y la forma de recolección de la información se realizó mediante encuesta, utilizando un cuestionario estructurado que lo responden los jefes de sistemas o informática y jefes de personal o de recursos humanos y, lo devuelven mediante correo electrónico o fax.

Se seleccionó una muestra probabilística utilizando muestreo aleatorio simple con una asignación proporcional de la muestra al número de empresas en Bogotá, Medellín, Cali, Popayán, Barranquilla, Bucaramanga y Manizales.

En las instituciones de formación y capacitación se consideran tres grupos: el nivel universitario formado por los programas de pregrado y postgrado, el nivel intermedio profesional en el cual se brinda formación técnica y tecnológica y las instituciones de educación no formal.

\section{Tamaño de la muestra}

\section{Empresas usuarias}

El universo de empresas usuarias lo constituyen 321.099 registradas en las Cámaras de Comercio de las ciudades de la cobertura geográfica, en las 9 actividades económicas y consolidadas por Confecámaras en 1998. Cada una de las 7 ciudades constituye un estrato.

Se escogió una muestra aleatoria simple con un 95\% de confianza teniendo en cuenta cada uno de los valores de los porcentajes o proporciones de las 4 preguntas más importantes; el mayor tamaño de la muestra para esas 4 preguntas ocurre en el $24 \%$; se calcula el tamaño de la muestra con la siguiente fórmula:

$$
o=(\text { p.qz2 }) / \text { e2 donde, }
$$

$P:$ porcentaje de empresas que consideran que es indispensable un conocimiento en sistemas de información: $p=24 \%$.

Q: es el porcentaje de empresas que no consideran indispensable esos conocimientos.

Z: cuantil de la distribución normal con un 95\% de confianza: $z=1.96$.

El error e, es la precisión deseada en la estimación del porcentaje: e $=6.5 \%$

$\mathrm{N}=$ es el número de empresas usuarias en las 7 ciudades: $\mathrm{N}=321.099$ empresas.

El 95\% de confianza significa que en 95 de cada 100 muestras que se tomen, el error en la estimación de este porcentaje, no supera el $6.5 \%$. 
El tamaño de la muestra $\mathrm{n}=167$ empresas; no se hace una corrección para poblaciones finitas porque $\mathrm{n} / \mathrm{N}$ es menor que el $5 \%$.

Para efectos de la recolección de la información en las 7 ciudades, se le asignó un tamaño de muestra proporcional al número de empresas en cada ciudad, en la siguiente forma:

Bogotá:

Medellín:

$167(154482 / 321099)=80$ empresas.

Cali: $167(43.011 / 321.099)=22$ empresas.

Barranquilla: $167(49.453 / 321.099)=26$ empresas

Bucaramanga:

Manizales:

Popayán:

$167(31.709 / 321.099)=16$ empresas .

$167(22.333 / 321.099)=12$ empresas.

$167(13.871 / 321.099)=7$ empresas.

$167(6.290 / 321.099)=4$ empresas.

La muestra específica por ciudad es importante porque posibilita un análisis regional, pero el reto, para darle validez al estudio, era lograr la consulta a las 167 empresas a escala nacional.

\section{Empresas proveedoras de tecnología}

La muestra más grande de las empresas proveedoras se obtiene para $p=65 \%$ con la misma fórmula utilizada para las empresas usuarias y con un error del $9 \%$; se obtiene un tamaño de muestra para las 7 ciudades de $n=76$ empresas proveedoras. Se considera que el número de empresas proveedoras en el país es $\mathrm{N}=1.000$ empresas; se hace la corrección del tamaño de la muestra para poblaciones finitas y el resultado es $n=71$ empresas.

\section{Organizaciones educativas}

Se seleccionó una muestra por conveniencia, en cada una de las ciudades que participan en el estudio, teniendo en cuenta su tradición en el ámbito regional o nacional en cada uno de los tres grupos: universitario, intermedio profesional y educación no formal

La unidad de análisis fue la institución, y la información la proporcionaron los decanos o los directores de los programas. Las variables más importantes son el perfil profesional, objetivos y plan de estudios.

\section{Instrumentos de recolección de la información}

Se elaboraron dos cuestionarios para recoger la información en las empresas usuarias y proveedoras del área tele-informática.

El cuestionario de las empresas usuarias tiene 20 preguntas que corresponden a variables de tipo cualitativo.

El número total de registros de las preguntas cerradas para cada empresa usuaria es el producto del número de variables por su respectivo número de modalidades y es igual a 1.218.

El cuestionario de las empresas proveedoras consta de 15 preguntas que representan variables cualitativas. 
La información de las instituciones de formación y capacitación, se recogió desde los plegables con los cuales hacen promoción las instituciones para sus programas 0 carreras, de la información que para tal fin colocan algunas de éstas instituciones en sus paginas Web y de la información directa que suministraron los decanos, directores de programa y vicerrectoras académicas y de la información que las instituciones universitarias tienen registrada en el lcfes.

\section{Alcance de los resultados}

Los resultados del estudio alcanzan un nivel descriptivo; la información es de corte transversal y fue recogida en el año 2000. La cobertura geográfica abarca las ciudades de Bogotá, Medellín, Cali, Barranquilla, Bucaramanga, Manizales y Popayán, por ser éstas los centros regionales más representativos del país.

Se logró la caracterización ocupacional construyendo tipologías de las empresas de acuerdo a la forma como respondieron a las preguntas; se analizaron mapas preceptuales de las empresas y las variables más importantes para el estudio.

La información que se recogió en este estudio se cruzó con la que se produjo en las consultas a expertos y con la que se ha venido produciendo en las fuentes secundarias.

\section{Plan de análisis}

Para lograr una caracterización de las empresas usuarias se construye una matriz de datos formada en las filas por las empresas y en las columnas por las 222 variables que contiene el cuestionario; para cada una se calcula su frecuencia y en especial se analizan las variables que tienen que ver con las 4 preguntas más importantes del estudio:

i. Los conocimientos específicos para operarios, técnicos, tecnólogos e ingenieros del área Teleinformática

ii. Habilidades y destrezas

iii. Capacidades y actitudes

iv. Las necesidades de conocimientos informáticos y de comunicaciones para todas las personas de la empresa.

Adicionalmente, se hizo un análisis factorial de correspondencia múltiple, $\mathrm{ACM}^{1}$, que es una técnica estadística descriptiva y multivariante utilizada en el análisis de datos y para sintetizar información.

El ACM permite pasar de unas variables categóricas originales a un pequeño número de nuevas variables o factores tales que sinteticen la información de las variables originales $^{2}$.

"El ACM consiste en la búsqueda de los factores más próximos (más correlacionados) con todos los grupos de modalidades o preguntas conjuntamente" ${ }^{3}$.

\footnotetext{
${ }^{1}$ Revista Simposio de Estadística, Análisis de Correspondencia Alain Morineau, CISIA, Francia 6 al 10 de junio de 1994. Bogotá.

${ }^{2}$ Ibid

${ }^{3}$ Ibid 
En el ACM hay que tener en cuenta que las variables (o modalidades) que son escogidas por más individuos, o sea que tienen un efectivo alto, aparecen cerca del origen porque no discriminan y las que tienen menor efectivo aparecen lejos del origen, son las que tienen mayor variabilidad y son importantes en el análisis.

En este estudio se procede a hacer un ACM para efectuar una selección de las variables de mayor contribución en la formación de los ejes. Como inicialmente se tienen 222, el objetivo es seleccionar un grupo menor, teniendo en cuenta también el respectivo valor del coseno cuadrado que nos permite verificar si la representación es buena porque esté cercana a 1 ó 0.

Las contribuciones acumuladas que también arroja el paquete SPAD.N dá una medida de la correlación entre la variable categórica y los ejes factoriales; de acuerdo a como sea esa contribución, se puede hablar de la correlación entre dos variables que formen un factor.

Se hacen tres aplicaciones del ACM y se observa que este grupo de variables corresponde a los siguientes temas del cuestionario: conocimientos específicos, competencias laborales, habilidades y destrezas, capacidades y actitudes y el dominio de estrategias de información por parte de tecnólogos. Los cuatro primeros temas forman parte de las cinco preguntas más importantes para los investigadores en este estudio.

Este último grupo de variables hace contribuciones a 5 factores de acuerdo a su mayor contribución y al coseno cuadrado; se hace una interpretación de ellos. Se analizan también las coordenadas de las modalidades para observar oposiciones entre las modalidades.

Aclaración:A veces parece que se utilizan indistintamente los términos, variables y modalidades; éstas últimas son las opciones de respuesta de la primera; ejemplo, la variable sexo tiene dos modalidades: hombre, mujer.

Un procedimiento similar se sigue para las empresas usuarias.

También se hace una clasificación de las empresas de acuerdo a estas variables para observar los perfiles de los grupos.

Los programas de educación teleinformática son consultados en las bases de datos del Icfes y sólo se contemplarán aquellos programas que están dentro de los marcos de legalidad.

Se tomó el contenido programático de cada una de las carreras e instituciones seleccionadas y se construyó una matriz por nivel, programa, institución, ciudad y región en la que se clasificaron los perfiles profesionales.

En cada programa y nivel (ejemplo: ingeniería de sistemas), se construye la matriz con cada una de las competencias laborales que explican un perfil y se cruzan con el total de los programas de la muestra para obtener la frecuencia en que el perfil profesional $u$ ocupacional se presenta en cada una de las instituciones.

Posteriormente, se suma el total de frecuencias de cada una de las competencias laborales o profesionales presentes en un programa determinado. 
El valor (frecuencia) de una competencia específica se divide perla frecuencia total de la sumatoria de todas las competencias presentes en el programa.

El valor porcentual obtenido al multiplicar el resultado de la división por cien determina la importancia cuantitativa de dicha competencia en el programa.

Con el procedimiento ilustrado se identifican las competencias presentes en cada uno de los programas y niveles y se clasifican en gráficas y tablas que permiten su comparación.

La clasificación anterior permite establecer la distribución geográfica de los programas en el país.

Con información suministrada por las bases de datos de Colciencias y el Icfes, actualizadas al 31 de diciembre de 1999, se establecen las líneas de investigación registradas en el Icfes y las investigaciones y proyectos de desarrollo financiados por el Programa ETI (electrónica, telecomunicaciones e informática).

\section{Conclusiones}

En el área de la teleinformática se identifican cinco factores críticos de los cuales depende en gran medida su desarrollo o estancamiento, aspectos que influyen de forma directa en la competitividad de las empresas:

- Conocimiento, experiencia y actitud positiva para el mercadeo y venta de productos y servicios en los ámbitos nacional e internacional.

- Investigación y desarrollo.

- Procesos de contratación.

- Profesionales y tecnólogos con alto nivel de formación y experiencia en gerencia de proyectos teleinformáticos y programación.

- Cultura de la calidad en el desarrollo de proyectos teleinformáticos y en la producción de software.

La sociedad globalizada reconoce como un hecho que se puede contar con ciencia y tecnología como ejes del desarrollo económico y social. Pero, ¿para qué nos puede servir (en Colombia) realizar su medición y disponer de indicadores o estadísticas?

Los alcances del estudio no permiten saber con precisión el caudal de actividades científicas y tecnológicas en el área, o la información obtenida nos muestra una pobre actividad y carencia de políticas e iniciativas serias y coordinadas.

Quedan algunas preguntas por resolver: ¿cuál es la contribución de la actividad científica y tecnológica al desarrollo social y económico, a la producción? ¿Cuánto se está invirtiendo y cuánto se debe invertir? ¿En qué se deben invertir los recursos destinados a la actividad científica y tecnológica? ¿Cómo conocer la real oferta que la ciencia y la tecnología deben proveer para satisfacer las necesidades básicas y productivas de la sociedad en el área teleinformática? ¿Con qué fin debemos medir la actividad científica y 
tecnológica teleinformática en Colombia? ¿Cómo lograr mayor preocupación y compromiso de la sociedad y del Estado en la investigación y desarrollo?

Por lo anterior, en materia de actividad científica y tecnológica, es menester concluir que existe una urgente necesidad de ampliar los conocimientos sobre las actividades y los procesos de desarrollo científico y tecnológico.

Colombia debe preocuparse más por conocer los problemas fundamentales, las brechas críticas de desarrollo científico y tecnológico y por prestar especial atención a este aspecto, y la sociedad entera debe esforzarse por tomar conciencia que el ignorarlo, es sinónimo de marginalidad y consumo desmedido de conocimiento científico y tecnológico, que finalmente tiene como consecuencia el empobrecimiento generado de la población y la pérdida de autonomía como Nación.

\section{Entorno organizacional}

El 7 de octubre de 1985, afirmaba el doctor Ricardo Quintana Sighinolfi, en el discurso inaugural del Primer Congreso de Informática realizado en la ciudad de Cartagena de Indias "La realización del Primer Congreso Colombiano de Informática es la culminación de una primera etapa dentro del proceso que están desarrollando las asociaciones de informática, ACCIO, ACIS y ACUC, para formar un sólo ente con las características que todos deseamos para que su voz sea oída, especialmente en los ámbitos gubernamentales, en aquellos temas que son de especial interés para el desarrollo informático del país"4.

Hoy, 15 años después, concluimos que el área teleinformática se encuentra acéfala. Las asociaciones privadas y los diferentes entes gubernamentales no han logrado interpretar con claridad las necesidades tecnológicas, sociales, económicas y políticas surgidas por desequilibrio de la cultura o como necesidad ideal de la sociedad globalizada.

Formalmente, el área teleinformática no existe y 30 años de representación de los ingenieros, usuarios y empresarios no han sido suficientes para lograr cooperación, acuerdo y trabajo colaborativo entre las agremiaciones privadas y el gobierno.

Contrario a lo que podría creerse, hemos de concluir que la atomización gremial del área teleinformática se constituye en uno de sus grandes obstáculos de desarrollo y de interlocución con el gobierno.

En la actualidad existen 7 gremios o asociaciones privadas que compiten entre sí la representación de los proveedores de tecnologías informáticas, los industriales del software, las empresas usuarias y los ingenieros de sistemas.

Las asociaciones privadas con mayor reconocimiento y tradición en el área teleinformática son ACIS, FEDESOFT y ACUC.

ACIS, agremia y representa a los ingenieros de sistemas. FEDESOFT, agremia y representa las empresas dedicadas a la producción de software y a la prestación de servicios informáticos.

\footnotetext{
${ }^{4}$ ACIS, ACCIO y ACUC. Memorias del $1^{\circ}$ Congreso Colombiano de Informátiao, Bogotá, Editorial Presencia, 1985, p. 7.
} 
ACUC, agremia y representa a las empresas usuarias de informática y comunicaciones.

\section{Tipos de empresas}

La falta de reconocimiento del área teleinformática como sector, imposibilita una clasificación que sea aceptada por los diferentes entes que la conforman y por sus actores y protagonistas.

El estudio conduce a clasificar las empresas en cuatro (4) grandes grupos:

- Industria del software.

- Empresas distribuidoras y comercializadoras de productos informáticos.

- Empresas proveedoras de acceso a Internet

- Empresas productoras y ensambladoras de hardware.

\section{Entorno económico}

Es casi imposible establecer la incidencia de los servicios y productos teleinformáticos en la economía nacional.

De los diferentes grupos de empresas se tienen cifras, más o menos confiables de la Industria del software y de los servicios relacionados con Internet.

\section{Industria del software}

En materia económica, las cifras que suministra el Banco de la República, el Ministerio de Comunicaciones y el Ministerio de Comercio Exterior son contradictorias y presentan grandes diferencias con relación a los datos suministrados por empresas consultoras de carácter internacional.

Ante el gobierno nacional no está definido con claridad si la industria del software es de servicio o de producto. Los industriales del software agremiados en FEDESOFT la definen como una industria de servicios.

La industria del software comprende dos elementos esenciales: el de bienes (programas en medio magnético) que cuentan con posición arancelaria definida, arancel e IVA y otro como servicio (profesional informático) el cual no tiene posición arancelaria y su registro se hace a través de estadísticas de servicio con base en encuestas.

La asesoría informática y la producción de software son hoy las actividades más importantes de la industria del software, y es de éstas de donde más se contribuye con la implernentación de una infraestructura computacional, indispensable para acceder a la llamada sociedad del conocimiento.

La industria nacional de software presenta una mayor actividad económica en la producción de software; sin embargo, es clara la tendencia de crecimiento del mercado de los servicios informáticos. Las empresas de la industria nacional que registran mayor crecimiento y se encuentran consolidadas, están dedicadas a la prestación de servicios profesionales informáticos en los sectores: industrial, financiero, comercio, comunicaciones y servicios públicos. 
Se estima que el promedio anual del crecimiento de la industria del software es del $16 \%$. Si esto llegase a cumplirse, para el año 2002 las ventas totales (de paquetes de software) sumarían más de US\$282 millones, y podrían generar de forma directa 7.681 empleos.

\section{Internet}

Colombia ocupa el $4^{\circ}$ lugar en América Latina en población conectada a Internet. Los ritmos de crecimiento son significativos.

El estudio pudo establecer que las empresas que tienen activos superiores a los $\$ 2.200$ millones, tienen conexión a Internet y aproximadamente un $87 \%$ tienen un sitio Web.

Cada año aumenta de forma significativa el registro de nombres punto co (.co). Si la tendencia registrada en los dos últimos años continúa, se estima que para diciembre 31 del presente año habrá más de 7.500 personas, organizaciones y empresas con su identificación y domicilio en Internet. co.

\section{Clasificación de las empresas teleinformáticas}

El $13 \%$ de las empresas cuentan con activos superiores a $\$ 2.200$ millones, el $29 \%$ con activos inferiores a $\$ 89$ millones y el $58 \%$ con activos inferiores a $\$ 2.200$ millones y superiores a $\$ 89$ millones.

El 3\% de las empresas del área teleinformática son unipersonales: el 5\% tienen más de 199 trabajadores, el 38\% 10 trabajadores y el 54\% tienen entre 11 y 199 trabajadores.

Según la antigüedad, las empresas se clasifican así: el 3\% menos de un año, el $90 \%$ más de 20 años, el $25 \%$ entre 11 y 20 años, el $33 \%$ entre 6 y 10 años y el $30 \%$ entre 1 y 5 años.

Los sectores económicos donde se presenta una mayor participación y competencia entre las empresas teleinformáticas son: comercio al por mayor y menor; establecimientos financieros, seguros e inmuebles; industria manufacturera; transporte, almacenamiento y comunicaciones; construcción; electricidad, gas y agua.

Los sectores donde se presenta un menor porcentaje de empresas compitiendo son el de canteras y las entidades del Estado.

La poca competencia y participación de un mayor número de empresas del área teleinformática en la venta de productos y servicios ante las entidades del Estado puede ser reflejo de monopolios en la contratación, de tráfico de influencias y de problemas asociados con la corrupción o de falta de preparación y conocimiento para concursar en licitaciones públicas.

\section{Caracterización del entorno educativo}

El $8 \%$ de la formación possecundaria, profesional y de posgrado son programas de informática y comunicaciones. 
El 8\% representa 514 programas distribuidos así:

\begin{tabular}{|l|c|c|}
\hline Tipo de programa & Total & $\%$ \\
\hline Licenciaturas & 15 & $2.9 \%$ \\
\hline Maestrías & 16 & $3.1 \%$ \\
\hline Ingeniería electrónica & 52 & $10.1 \%$ \\
\hline Técnicas & 71 & $13.8 \%$ \\
\hline Ingeniería de sistemas & 91 & $17.8 \%$ \\
\hline Tecnológicas & 130 & $25.3 \%$ \\
\hline Especializaciones & 139 & $27.0 \%$ \\
\hline Total & 514 & $100 \%$ \\
\hline
\end{tabular}

Aproximadamente el $36 \%$ de los programas de formación se encuentran concentrados en Bogotá y más del 65\% en las ciudades de Bogotá, Medellín y Cali.

En todos los niveles de formación se encuentra un alto énfasis (más del $40 \%$ del total de asignaturas) de formación en el área de ciencias básicas de ingeniería. El área de formación de menor énfasis en todos los programas es la de aplicación profesional.

Los programas de formación ofrecidos por las universidades e institutos concentran su atención en la formación y profundización profesional. Muy pocos adelantan proyectos de investigación o tienen estructuradas líneas o proyectos de investigación.

El área teleinformática es atendida por 187 instituciones de educación superior y postsecundaria, 14 de ellas cuentan con programas o proyectos de investigación en el Programa ETI. Esto equivale a que el $7 \%$ participa de proyectos financiados por Colciencias. El 14\%, que incluye a las anteriores, tienen líneas y proyectos de investigación registrados en el Icfes.

En el conjunto de instituciones de educación superior se encuentra una fuerte tendencia a ofrecer especializaciones y a través de éstas a participar de proyectos de consultaría informática, especialmente con entidades del Estado.

El alto número de especializaciones y la fuerte inclinación a la consultaría hace que se descuide y desatiendan los proyectos de investigación.

Dentro de las universidades que cuentan con proyectos de investigación y desarrollo financiados por Colciencias (Programa ETI) se encuentra que el $64,29 \%$ son oficiales o del Estado y el $35,71 \%$ privadas.

A pesar de ser las universidades del Estado las que más aportan y participan de la investigación en el área teleinformática, los gobiernos vienen reduciendo de forma paulatina y significativa su cobertura en la educación. Hoy el $30 \%$ de la educación superior es atendida por instituciones de carácter oficial y el $70 \%$ por instituciones privadas que poco participan o contribuyen con la investigación en el área teleinformática.

\section{Perfil ocupacional}

El estudio de campo (encuesta a proveedores y usuarios) muestra una gran preocupación de los empresarias del sector usuario y de las empresas proveedoras por 
mejorar la formación de los ingenieros, tecnólogos y técnicos en cuanto a sus habilidades para el autoaprendizaje; disposición para el trabajo en grupo y colaborativo; disposición y actitud para la comunicación interpersonal y social.

En general los empresarios consideran que sus empresas y áreas de informática lograrían un gran avance y progreso si sus ingenieros, técnicas y tecnólogos alcanzan mayores niveles y compromisos en su disposición para mejorar el servicio.

Con base en los resultados del estudio se puede afirmar que las empresas proveedoras de servicios y productos informáticos requieren altos niveles de formación en:

- Desarrollo de software.

- Comercialización de productos y servicias informáticos.

- Gerencia de proyectas informáticas.

Los empresarias consideran, según su experiencia y conocimiento, que los ingenieros, tecnólogos y técnicos del área teleinformática deben tener o contar can los siguientes conocimientos, habilidades, destrezas y actitudes para que sus empresas sean más competitivas:

\section{Ingenieros:}

Capacidad para el trabajo en grupo; capacidad para realizar los objetivos y tareas propuestas; habilidades y destrezas para el trabajo colaborativo; capacidad creativa; capacidad de sugerir y diseñar soluciones; capacidad y estrategias para la administración del tiempo; capacidad de adaptación al medio; capacidad de autoaprendizaje; disponibilidad al cambio; capacidad y habilidades para el manejo de la comunicación oral y escrita.

\section{Tecnólogos:}

Capacidad creativa; capacidad y conocimiento para el diseño y desarrollo de proyectos; capacidad para el trabaja en grupo; capacidad y habilidades para las relaciones interpersonales e interinstitucionales; capacidad y habilidades para la administración del tiempo; dominio en la comunicación oral y escrita; capacidad de realizar los objetivas y tareas propuestas; capacidad para sugerir y diseñar soluciones; disponibilidad al cambia disponibilidad para el trabaja colaborativo; capacidad de adaptación al medio; capacidad de autoaprendizaje; capacidad de análisis y síntesis; habilidad de producir y llevar la información conforme a los parámetros acordadas can el usuaria; capacidad crítica y reflexiva; capacidad para establecer analogías y modelar conocimiento; capacidad de negociación; capacidad de liderazgo; capacidad de interpretación y adaptación al entorno institucional del usuario a quien se le venden los productas y servicias y, conocimientos en: Integración y administración de sistemas o procesos de información; implementación de procesos de información; desarrolla de sistemas a usar en el procesamiento de información; determinación y características del conocimiento que debe transmitir la información; programación y desarrollo de software; manejo y conocimiento en los aplicativos de oficina y conocimiento de las normas de calidad. 
Técnicos:

Capacidad para el diseño y desarrollo de proyectas; capacidad crítica y reflexiva; capacidad creativa; capacidad de relaciones interpersonales e interinstitucional; capacidad de análisis y síntesis; dominio en la comunicación oral y escrita; capacidad para realizar los objetivos y las tareas propuestas.

Independiente de si trabajan para empresas usuarias a proveedoras, los ingenieros y tecnólogos se ocupan principalmente en las siguientes actividades económicas:

- Consultaría y desarrollo de software.

- Asesoría y consultoría de hardware y software.

- Venta, implementación y asesaría de sistemas de gestión empresarial.

- Comercio y venta de hardware.

- Diseño y desarrollo de portales y sitias Web.

- Desarrollo de software especializado y a la medida.

- Asesoría, implementación y desarrollo de sistemas de información integrales y globalizados.

- Diseño y desarrollo de soluciones integrales informáticas.

- Implantación de procesas de información.

- Soporte técnico a equipos y software.

- Soporte y mantenimiento a redes

-Administración de bases de datos. 